\title{
A New Solution for the Dispersive Element in Astronomical Spectrographs
}

\author{
Harland W. Epps, ${ }^{1}$ Judith G. Cohen, ${ }^{2}$ And J. Christopher Clemens ${ }^{3}$ \\ Received 2009 August 3; accepted 2009 December 7; published 2010 January 26
}

\begin{abstract}
We present a new solution for the dispersive element in astronomical spectrographs that, in many cases, can provide an upgrade path to enhance the spectral resolution of existing moderate-resolution reflectiongrating spectrographs. We demonstrate that in the case of LRIS-R at the Keck 1 Telescope, a spectral resolution of 18,000 can be achieved with reasonable throughput under good seeing conditions.
\end{abstract}

\section{INTRODUCTION}

We have been carrying out a preliminary design study to explore achieving multiplexing of high-resolution optical spectroscopy for point sources at the Keck Observatory. This effort arose from our desire to provide capabilities for the Keck user community comparable to those offered by the successful fiber-optic-fed instruments FLAMES + GIRAFFE and FLAMES + UVES (Pasquini et al. 2002; Dekker et al. 2000). These multiplex up to 130 targets to the medium-high resolution optical GIRAFFE spectrograph, with up to 8 targets to the VLT's high-resolution UVES spectrograph. These instruments have made major advances possible in a number of areas, including near-field cosmology and Galactic stellar spectroscopy. Since the cost of building a new instrument for the Keck Observatory for this purpose is in excess of 15 million dollars, we explored the possibility of modifying an existing moderatedispersion multiobject spectrograph to achieve this goal in a way that also preserves the present mode of operation of the spectrograph.

In the course of this effort, we found what we believe to be a new solution for the dispersive element in astronomical spectrographs. It substantially enhances the spectral resolution of suitable existing moderate-resolution spectrographs. Our solution can thus provide a path for upgrading and augmenting the capability of many existing instruments. It will be economical and, in most cases, fairly easy to implement. In the case of new spectrographs, we believe adopting this solution for the disperser, instead of a conventional reflection grating, can result in the design of smaller, cheaper instruments that can achieve a given specified high spectral resolution.

\footnotetext{
${ }^{1}$ UCO/Lick Observatory, University of California, Santa Cruz, CA 95064; epps@ucolick.org.

${ }^{2}$ Palomar Observatory, California Institute of Technology, Pasadena, CA 91125; jlc@astro.caltech.edu.

${ }^{3}$ Department of Physics and Astronomy, University of North Carolina, Chapel Hill, NC 27599-3255; clemens@physics.unc.edu.
}

\section{EXISTING SOLUTIONS}

Some efforts to increase the spectral resolution of an existing multiobject spectrograph have involved inserting an echelle grating and using the existing reflection grating (or a prism) as a cross disperser. This concept was included in the design of the Norris Spectrograph for the $5 \mathrm{~m}$ Hale Telescope at Palomar Mountain (Hamilton et al. 1993) but the echelle mode was never implemented, due to lack of funds. To maintain object multiplexing in such a spectrograph, the number of echelle orders to be used must be limited by interference filters to be a small number.

The multiobject high-resolution echellete addition to IMACS on the Magellan $6.5 \mathrm{~m}$ Telescope is a variant of this scheme; a prism cross-dispersed echellete grating is now available as an optional module on the moderate-resolution IMACS instrument (Sutin \& McWilliam 2003). The echellette requires 10 orders for full spectral coverage over the optical regime; a filter can be used to restrict the wavelength range retained and hence to increase the multiplexing capability.

Unfortunately as the telescope diameter increases beyond that of the $6.5 \mathrm{~m}$ Magellan Telescopes, this upgrade path becomes more difficult, and we have established that it is not practical for the two Keck multiobject moderate-resolution optical spectrographs, LRIS (Oke et al. 1995) and DEIMOS (Faber et al. 2000).

Other techniques that can be used to try to achieve higher spectral resolution include increasing the line density or using a reflection grating nominally designed for use in the first order near IR, to cover optical wavelengths in the second order instead. While this doubles the spectral dispersion, it also leads to very large angles of incidence and large anamorphic factors. The anamorphism reduces the projected slit width, which can lead to inadequate pixel sampling. The large angle of incidence and hence large projection factor also demands a grating with one dimension much larger than the beam size, which is typically $\sim 150 \mathrm{~mm}$ in many optical spectrographs. If the grating does not have the required width in the direction of the dispersion, a severe vignetting light loss will occur. A suite of $154 \times$ $206 \mathrm{~mm}$ gratings is available from the Richardson Grating 
Laboratory of the Newport Corporation, but larger ones are not readily available. Large anamorphic factors also place added demands on the camera's entrance aperture and optical design, which are not normally satisfied in moderate-resolution spectrographs.

High-quality reflective gratings with rulings exceeding 1200 lines $\mathrm{mm}^{-1}$, suitable for first-order use at optical wavelengths and in sizes large enough for a $150 \mathrm{~mm}$ diameter beam, are not commercially available to our knowledge. Ruling a new master for such a high-resolution grating would be very expensive, and would require a minimum timescale of a year. There is only one commercial source for such a large astronomical grating in the US today, the Richardson Grating Laboratory, now part of Newport Corporation.

\section{OUR NEW SOLUTION}

Our solution involves replacing the normal low-order reflection grating with a pair of volume phase holographic (VPH) gratings used in transmission. The use of a pair of VPH gratings enables bending the beam so that the axial light path matches that of a normal reflection-grating spectrograph with an included angle $Q$ between the collimator and camera optical axes. VPH gratings, unlike reflection gratings, are currently available from several commercial vendors in the required (large) sizes, with much higher line densities, for a price less than $\$ 20,000$ each. The timescale for acquiring such VPH gratings is considerably shorter than that for ruling a new master for a large reflection grating.

The two identical VPH gratings in the pair form a "tent," with both ends open, whose entrance and exit are identical isoceles triangles and whose sides are each a VPH grating, as shown in Figure 1. The apex angle of the isoceles triangle $(A)$ is fixed at a value of $(90.0-(Q / 2))$ so that light from the collimator at the desired central wavelength passes through the VPH pair and ends up heading toward the camera when the diffraction of each VPH is taken into account. If the VPH pair is to be used to upgrade an existing spectrograph, to allow a higher-resolution mode, the included angle $Q$ between the collimator and camera optical axes is fixed and is determined by the design of the existing instrument.

Once the apex angle $A$ is known, there is only one free design parameter for the dispersive element, the line density of the VPH grating pair, $N_{l}$ lines $\mathrm{mm}^{-1}$. Given the fixed included angle $Q$, the dispersion (in radians $\mu^{-1}$ ) is proportional to $N_{l}$ of the VPH gratings while the central wavelength is proportional to $N_{l}^{-1}$. For a given slit width, the spectral resolution is independent of $N_{l}$. The spectral coverage is then limited, when this is used as an upgrade to an existing instrument, by the width of the detector, which sets the extent of the first order that fits across the detector. Figure 2 shows a layout for a VPH pair in the Red arm of the existing LRIS spectrograph at the Keck 1 Telescope. Some performance quantities are tabulated in Table 1 for the double-VPH disperser mode in an upgraded LRIS-R.

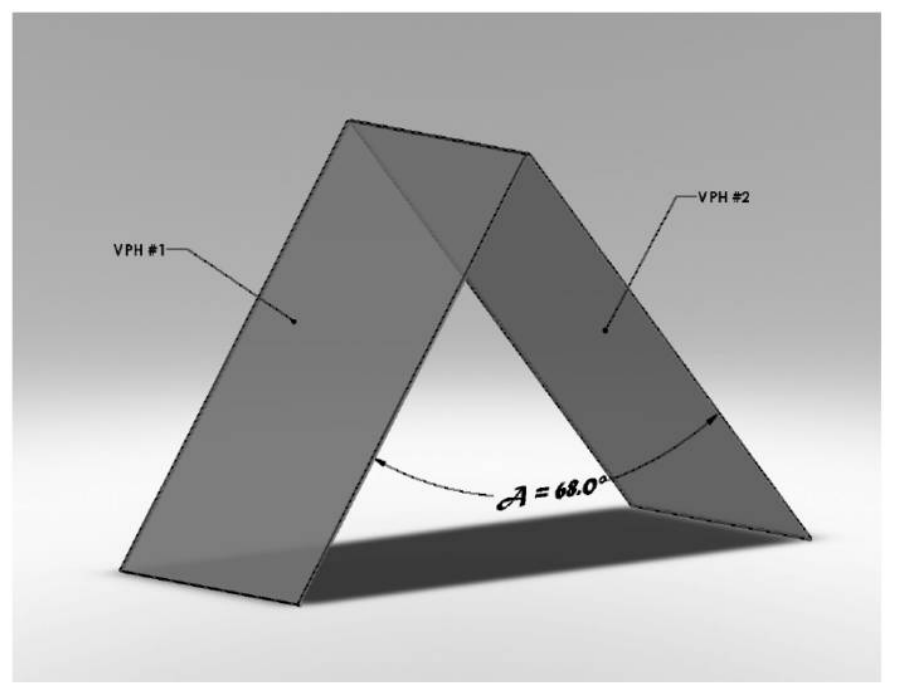

FIG. 1.-Sketch of a pair of VPH gratings assembled into a "tent" to function as a disperser.

VPH gratings to accommodate a $\sim 150 \mathrm{~mm}$ beam can be manufactured with $N_{l} \sim 3000$ lines $\mathrm{mm}^{-1}$, allowing the possibility of reaching much higher spectral resolution than in a normal first-order reflection-grating spectrograph, for the same projected slit width. The gain in spectral resolution over a 1200 lines $\mathrm{mm}^{-1}$ reflection grating used in first order in a normal astronomical spectrograph is then $\approx 2 N_{l} /[1200 \cos (A / 2)]$ when a VPH pair is used as described here.

\subsection{Advantages}

The advantages of the proposed double-VPH disperser are many. There are no moving or tiltable parts. Mechanical flexure, the bane of many existing spectrographs, is easier to control as a VPH pair is considerably lighter than a reflection grating for the same beam size and the former does not have to be rotated. VPH gratings, at least up to sizes of $\sim 205 \mathrm{~mm}$ on a side, are readily available at "reasonable" prices.

If the existing spectrograph to be modified has multiobject capability, which is more common in moderate-resolution instruments than in high-resolution ones, this capability is unaffected by the introduction of the double-VPH grating as the dispersive element; the instrument will perform in the same way when the dispersive element is changed to the VPH pair, making this an ideal upgrade path.

Furthermore, adding the option of a VPH pair to a moderateresolution multiobject spectrograph enables the use of both modes, provided that a switch between the two types of dispersers is implemented. Thus both can take advantage of the multiplexing already incorporated into the moderate-resolution instrument.

For a particular pair of VPH gratings, the central wavelength and spectral range in each order are fixed by $N_{l}$ once the 


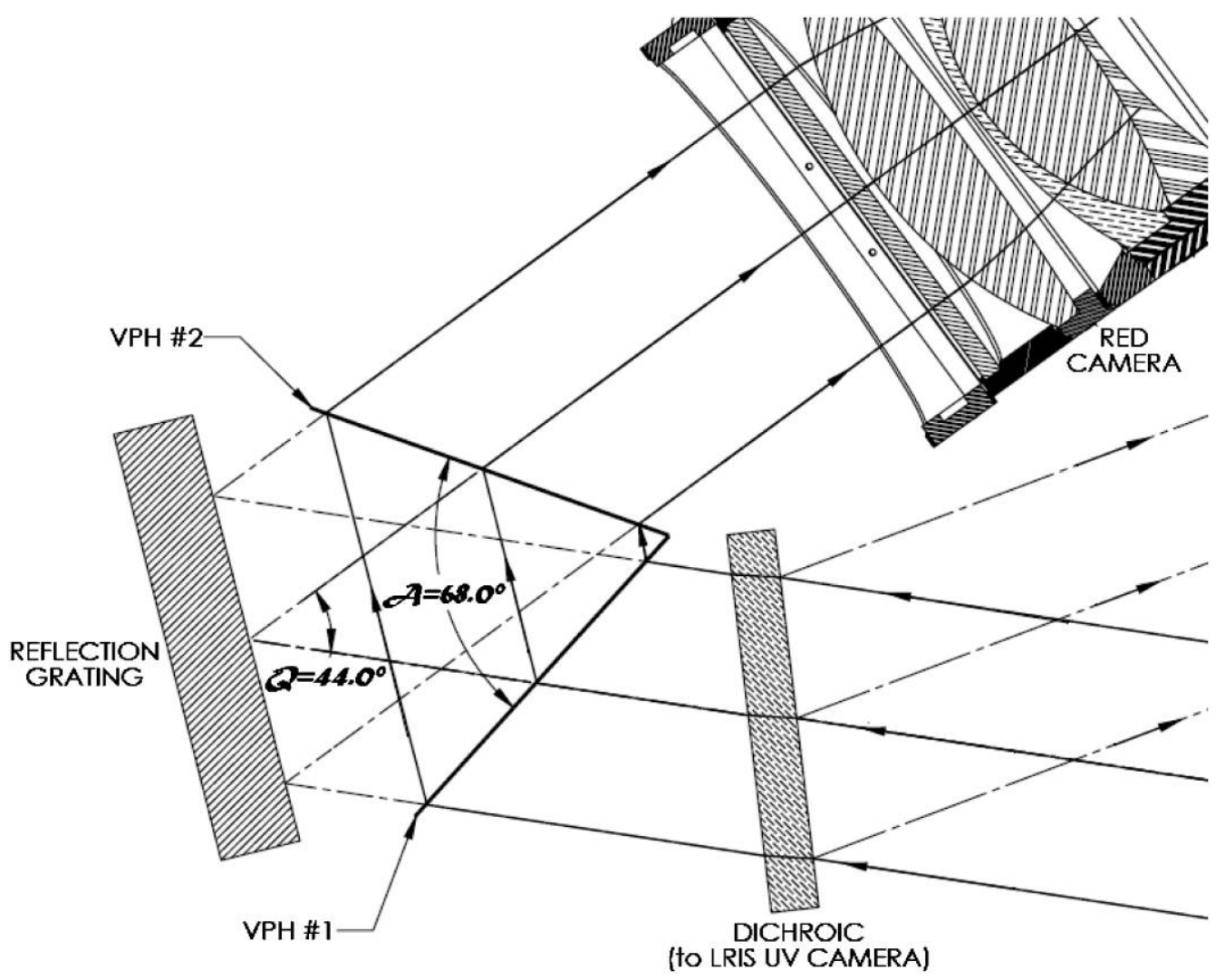

FIG. 2.-Region around the reflection grating used as a disperser in the moderate-resolution LRIS spectrograph at the Keck 1 Telescope. The placement of the proposed VPH pair upgrade to achieve higher spectral resolution while maintaining the multiplexing capability of LRIS-R is indicated.

included angle $Q$ between the collimator and camera optical axes is specified, either in a new design or an as-built design. Given the relatively short wavelength regime a given VPH pair must cover, it is not difficult to make their throughput comparable, for the pair, to that of a conventional reflection grating intended for use over a much broader wavelength regime. Quantitative details are given in $\S 4.1$.

TABLE 1

Properties of VPH Pairs in LRIS-R at the 10 M Keck 1 Telescope

\begin{tabular}{|c|c|c|c|}
\hline $\begin{array}{l}\text { Lines mm }{ }^{-1} \text { of } \\
\text { VPH pair }\end{array}$ & $\begin{array}{l}\text { Central } \lambda \\
(\AA)\end{array}$ & $\begin{array}{l}\text { Dispersion } \\
\left(\text { radians } \mu^{-1}\right)\end{array}$ & $\begin{array}{c}\text { Spectral coverage (LRIS-R) } \\
(\AA)\end{array}$ \\
\hline 1100 & 10167 & 2.6537 & 759 \\
\hline $1200 \quad \ldots \ldots \ldots$ & 9320 & 2.8949 & 696 \\
\hline $1300 \quad \ldots \ldots \ldots$ & 8603 & 3.1362 & 642 \\
\hline $1500 \quad \ldots \ldots \ldots$ & 7456 & 3.6187 & 557 \\
\hline $1700 \quad \ldots \ldots \ldots$ & 6579 & 4.1011 & 491 \\
\hline $1900 \quad \ldots \ldots \ldots$ & 5886 & 4.5836 & 439 \\
\hline 2100 & 5326 & 5.0661 & 398 \\
\hline $2300 \quad \ldots$ & 4863 & 5.5486 & 363 \\
\hline $2500 \ldots$ & 4474 & 6.0311 & 334 \\
\hline $2700 \quad \ldots \ldots \ldots$ & 4142 & 6.5136 & 309 \\
\hline $2900 \quad \ldots \ldots \ldots$ & 3857 & 6.9961 & 288 \\
\hline
\end{tabular}

${ }^{a}$ Section 4 gives the relevant characteristics of LRIS-R and its detector.
In the design of astronomical spectrographs, aberrations must be balanced, and the final image often suffers from some degree of axial chromatic aberration. Since the spectral range within which a VPH pair will be used is relatively small (limited by the detector width), the axial chromatic aberration can largely be eliminated for a given setup by optimizing the spectrograph focus for each VPH pair.

\subsection{Disadvantages}

There are of course disadvantages as well. The major one is the limited spectral range that can be covered by a given VPH pair due to the fixed collimator-to-camera included angle $Q$. Given this, several such VPH pairs will need to be purchased, each of which covers a spectral window of particular interest. Such a scheme is best suited for stellar work where the redshift is fixed at $z \sim 0.0$. The variety of redshifts of extragalactic sources makes it impossible to optimize and limit the number of wavelength ranges that must be covered. Full coverage of redshift space using VPH gratings would be costly, but not nearly as expensive as building a new high-resolution multiplexing spectrograph from scratch.

Ideally the required pairs of VPH gratings for most/all observations can all be accommodated within a slide or storage 
box plus changer mechanism, designed with the safety of the instrument optics as well as ease of use in mind. This would make it possible for all the VPH grating pairs that are to be used in a given observing run to be installed within the instrument, and the pair in use could be switched during the night via remote control. If there are more VPH pairs in regular use than can be accommodated by such a mechanism, manual switching would be necessary. This is undesirable as such manual changes would become a maintenance issue and affect the long-term operational cost. There is also a concern for the safety of the other optical components in the spectrograph during any procedure that requires manual access to its interior.

Implementation of the VPH pair within an existing instrument requires that the area around the reflection grating be fairly open and accessible so that a holder for the VPH grating pairs can be installed, ideally with a changer mechanism. This mechanism might be as simple as a manually-driven (or better yet, a remotely-driven) slide of VPH pairs. Some existing spectrographs contain other components, which occupy the desired volume for other key purposes, making it difficult or impossible to implement double-VPH gratings as an upgrade path. In addition, there is a requirement that the volume to be occupied by the VPH pair, which extends toward both the collimator and the camera from the nominal position of a reflection-grating disperser (see Fig. 2), does not include any part of the optical path that would obscure the beam in part or completely.

\section{PREDICTED LRIS-R PERFORMANCE COMPARISON}

The LRIS-R camera has recently been upgraded with two $(2048 \times 4096 \times 15 \mu)$ fully-depleted high-resistivity thick redsensitive CCDs from Lawrence Berkeley Laboratory (Stover et al. 2009). The measured LRIS-R final image scale on the Keck 1 Telescope is $0.135^{\prime \prime}$ pixel $^{-1}$ (Rockosi et al. 2009), which is consistent with the camera's original $305.0 \mathrm{~mm}$ effective focal length (Oke et al. 1995) even though a new field flattener lens was installed during the upgrade.

A 1200 line $\mathrm{mm}^{-1}$ reflection grating working in first order at a central wavelength of $5886.2 \AA$ is selected for detailed comparison with the 1900 line $\mathrm{mm}^{-1}$ double-VPH example in Table 1 . The reflection-grating setup produces a rather extreme anamorphic factor $(r=1.3993)$ and a finer ruling is not practical for reasons described in $\S 2$. A larger anamorphic factor would cause excessive vignetting and would lead to inadequate image quality, as it would overdrive the camera's optical design. Thus near-maximum reflection-grating resolution is expected in this example.

The comparison must be made with the same effective slit width(s) in pixels at the CCD, for both modes. Anamorphic demagnification will enable the slit to be wider in arcsec by the anamorphic factor $(r)$ relative to the double-VPH mode, which has no anamorphism. The quantitative comparison is given in Table 2.
Table 2 is not extended further because the spectral resolution would tend to become pixel-sampling limited; residual aberrations in the camera would limit the resolution as well. The effective slit-width limit should be determined experimentally but practical experience suggests that about 3.0 pixels will be a close approximation.

At a given pixel sampling, the wider slit in reflection-grating mode will tend to favor its throughput. However, that advantage will tend to disappear with better "seeing," and vignetting at the camera's entrance aperture due to the large anamorphism will cause light loss toward the ends of the spectrum as well. Thus we assert that it will be quite feasible to produce photonefficient multiplexed spectra at resolutions of $R=18,000$ or more with our proposed double-VPH disperser(s), used in LRIS-R with good "seeing." The fact that double-VPH resolution is independent of central wavelength is an important added advantage not shared by the reflection-grating mode.

\subsection{Efficiency of the Double-VPH Disperser}

We have used rigorous coupled wave analysis (RCWA) to investigate the efficiency of VPH gratings used in a doubleVPH mode. The Fresnel losses at the four air/glass boundaries have been neglected. In total they would be $\sim 1 \%$ depending on the details of the coatings over each of the narrow passbands. Figure 3 shows calculations for a pair of 1900 line $\mathrm{mm}^{-1}$ gratings optimized for use at an incident angle of $34^{\circ}$, which is appropriate for LRIS-R. The film thickness used in this model is $4.5 \mu$, and the index modulation is 0.07 , both of which are easily achievable by any of the VPH grating vendors. The upper curve shows the efficiency $(\epsilon)$ in single pass, over the spectral range that is covered by the recently upgraded LRIS-R detector. Light at the Bragg condition (5886 $\AA$ for this line density) is diffracted most efficiently and hits the second grating also at the Bragg condition, so the total efficiency is simply $\epsilon^{2}$. However, incident light at wavelengths different from the Bragg condition is not only diffracted less efficiently by the first grating, but also strikes the second grating even further from the Bragg condition because of the angular deviation introduced by the dispersive effect of the first grating. The penalty for this angular deviation is higher than for the mismatch to the Bragg wavelength, so the efficiency drops more steeply than $\epsilon^{2}$ at the off-Bragg

TABLE 2

Resolution Comparison For Optimal ReFLECTION-GRating Mode versus DOUble-VPH DISPERSION MODE IN LRIS-R

\begin{tabular}{|c|c|c|c|c|}
\hline $\begin{array}{l}\text { Slit Width } \\
\text { (pixels) }\end{array}$ & $\begin{array}{l}\text { Reflection Grating } \\
\text { (arcsec) }\end{array}$ & Resolution & $\begin{array}{l}\text { Double VPH } \\
\quad(\operatorname{arcsec})\end{array}$ & Resolution \\
\hline 7.0 & 1.32 & 2,052 & 0.95 & 7,837 \\
\hline $6.0 \ldots \ldots$ & 1.13 & 2,394 & 0.81 & 9,143 \\
\hline $5.0 \quad \ldots \ldots$ & 0.94 & 2,872 & 0.68 & 10,972 \\
\hline $4.0 \quad \ldots \ldots$ & 0.76 & 3,591 & 0.54 & 13,715 \\
\hline $3.0 \quad \ldots \ldots$ & 0.57 & 4,787 & 0.41 & 18,287 \\
\hline
\end{tabular}




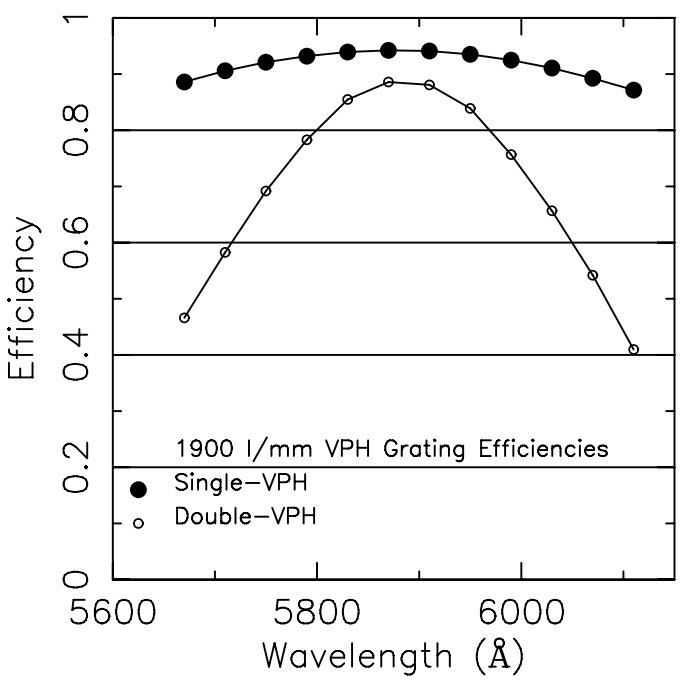

FIG. 3.-Efficiency of a single 1900 line $\mathrm{mm}^{-1}$ VPH grating is shown, as is that of a pair of them designed for use in LRIS-R. The wavelength coverage here and in Figs. 4 and 5 is limited by the CCD detector width.

wavelengths, as illustrated by the lower curve. The efficiency averaged over the $440 \AA$ wide spectral range is still quite high, $\sim 70 \%$.

In addition to understanding the efficiency of the 1900 line $\mathrm{mm}^{-1}$ grating, we have used RCWA to investigate whether the entire grating complement listed in Table 1 can be made at similar high efficiencies. We have modeled a family of gratings, all of which have 0.07 index modulation, but with thicknesses that vary more or less in inverse proportion to the line density. Figures 4 and 5 show the double-VPH efficiency curves for the

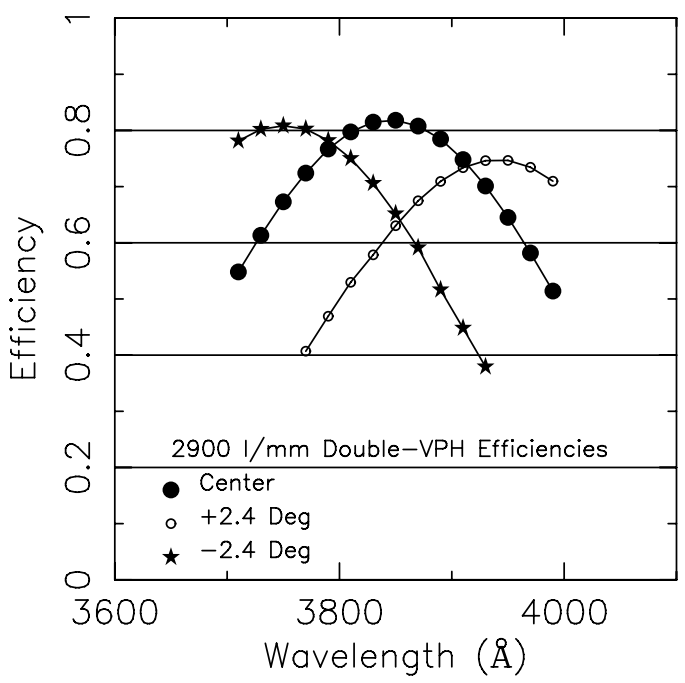

FIG. 4.-Efficiency of a pair of VPH gratings with 2900 lines $\mathrm{mm}^{-1}, 2.5 \mu$ thickness, and 0.07 index modulation is shown. Curves are also shown for efficiencies and wavelength coverage for objects that illuminate the first grating at $\pm 2.4^{\circ}$ from the $34^{\circ}$ central angle, as happens in multislit mode extreme cases.

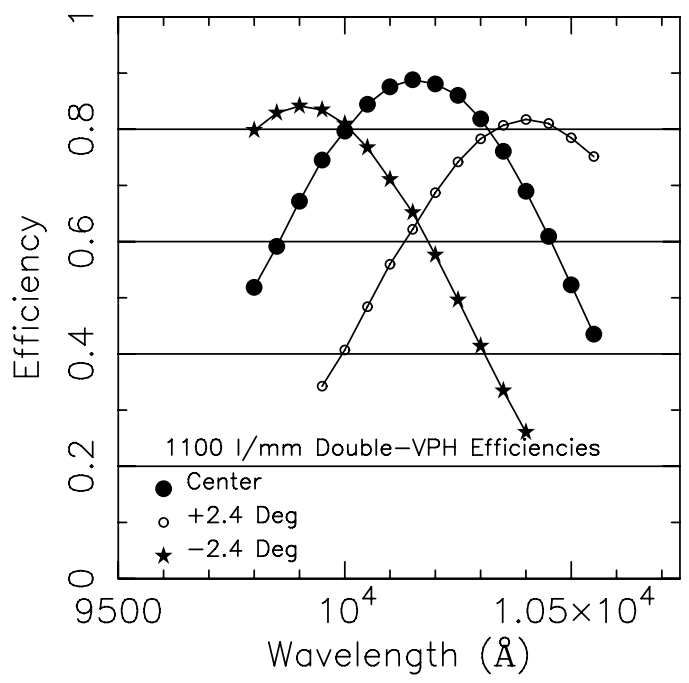

FIG. 5.-Same as Fig. 4, but for a pair of VPH gratings with 1100 lines $\mathrm{mm}^{-1}$, $7.5 \mu$ thickness, and 0.07 index modulation.

highest and lowest line density grating models, also over the wavelength range that can be detected in each case with LRIS-R. The most difficult grating to fabricate is that with 2900 lines $\mathrm{mm}^{-1}$ because it is only $2.5 \mu$ thick. Blanche et al. (2004) have published confirmation that CSI/ATHOL can make efficient gratings of $2.9 \mu$ thickness, but they have not confirmed this for high line density. However, during the preparation of this article, we were able to produce a sample in the Goodman Laboratory that has 3100 lines $\mathrm{mm}^{-1}$, a thickness of $2.5 \mu$, and a modulation of 0.07 , nearly identical to the grating modeled in Fig. 4.

It is also important to model $\epsilon$ for incident light that strikes the first grating off-axis. This can occur because LRIS is a multiobject spectrograph. Slitlets at extreme angles away from the center line of the slit mask in the dispersion direction, illuminate the grating at angles $\pm 2.4^{\circ}$ from the central angle. Figures 4 and 5 include curves showing this effect for slitlets displaced at these extreme locations. The peak efficiency is shifted from that of the on-axis curve and the mean efficiency is somewhat lower, although it averages $\sim 65 \%$ as shown. The spectral coverage is shifted as well, depending on the distance of the slitlet off of the center line of the slit mask, an effect that always occurs in multislit spectrographs.

We conclude from these representative calculations that double-VPH dispersers can be expected to provide average efficiencies of $\sim 65 \%$ to $70 \%$ over the entire complement of grating pairs and the full range of multislit locations available to LRIS-R.

The authors acknowledge partial support from a grant of seed money from the Keck Observatory to J. Cohen, H. Epps, and R. M. Rich. We thank R. M. Rich for his long-term advocacy for multiplexed high-resolution spectroscopy at the Keck Observatory. 


\section{REFERENCES}

Blanche, P. A., Gailly, P., Habraken, S., Lemaire, P., \& Jamar, C. 2004, Proc. SPIE, 5494, 208

Dekker, H., et al. 2000, Proc. SPIE, 4008, 534

Faber, S. M., et al. 2003, Proc. SPIE, 4841, 1657

Hamilton, D., et al. 1993, PASP, 105, 1308

Oke, J. B., et al. 1995, PASP, 107, 375
Pasquini, L., et al. 2002, Messenger, 110, 1

Rockosi, C., et al. 2009, LRIS-R Upgrade Commissioning Report, draft June 16, 2009

Stover, R., et al. 2004, Proc. SPIE, 5499, 518

Sutin, B. M., \& McWilliam, A. 2003, Proc. SPIE, 4841, 1357 